\title{
Risk of surgery in patients with stricturing type of Crohn's disease at the initial diagnosis: a single center experience
}

\author{
Yuji Maehata ${ }^{1,2}$, Yutaka Nagata ${ }^{1}$, Tomohiko Moriyama ${ }^{1}$, Yuichi Matsuno ${ }^{1}$, Atsushi Hirano ${ }^{1}$, Junji Umeno ${ }^{1}$, \\ Takehiro Torisu ${ }^{1}$, Tatsuya Manabe ${ }^{3}$, Takanari Kitazono ${ }^{1}$, Motohiro Esaki ${ }^{1,4}$ \\ ${ }^{I}$ Department of Medicine and Clinical Science, Graduate School of Medical Sciences, Kyushu University, Fukuoka; ${ }^{2}$ Maehata Clinic, Kagoshima; \\ ${ }^{3}$ Department of Surgery and Oncology, Graduate School of Medical Sciences, Kyushu University, Fukuoka; ${ }^{4}$ Department of Endoscopic \\ Diagnostics and Therapeutics, Saga University Hospital, Saga, Japan
}

Background/Aims: It remains uncertain which patients with stricturing-type Crohn's disease (CD) require early small bowel surgery after the initial diagnosis. We aimed to clarify clinical characteristics associated with the intervention in such condition of CD. Methods: We retrospectively evaluated the clinical course of 53 patients with CD and small bowel strictures who were initially treated with medications after the initial diagnosis. We investigated possible associations between small bowel surgery and the following: clinical factors and radiologic findings at initial diagnosis and the types of medications administered during follow-up. Results: Twenty-eight patients (53\%) required small bowel resection during a median follow-up period of 5.0 years (range, $0.5-14.3$ years). The cumulative incidence rates of small bowel surgery at 2,5 , and 10 years were $26.4 \%, 41.0 \%$, and $63.2 \%$, respectively. Univariate analysis indicated that obstructive symptoms $(P=0.036)$, long-segment stricture $(P<0.0001)$, and prestenotic dilation $(P<0.0001)$ on radiography were associated with small bowel surgery, and immunomodulatory $(P=0.037)$ and biological therapy $(P=0.008)$ were significant factors during follow-up. Multivariate analysis revealed that long-segment stricture (hazard ratio [HR], 4.25; 95\% confidence interval [CI], 1.78-10.53; $P=0.001$ ) and prestenotic dilation (HR, 3.41; 95\% CI, 1.24-9.62; $P=0.018$ ) on radiography showed a positive correlation with small bowel surgery, and biological therapy (HR, 0.40 ; 95\% CI, 0.15-0.99; $P=0.048$ ) showed a negative correlation. Conclusions: CD patients with long-segment stricture and prestenotic dilation on radiography seem to be at a higher risk of needing small bowel surgery. For such patients, early surgical intervention might be appropriate, even at initial diagnosis. (Intest Res 2019;17:357-364)

Key Words: Crohn disease; Stricturing type; Intestine, small; Surgery; Biological therapy

\section{INTRODUCTION}

Crohn's disease (CD) is a chronic inflammatory bowel disease of unknown etiology. Although CD involves any part of the GI tract, its intractable disease course and pathognomonic transmural inflammation often cause intestinal complications, especially in the small bowel. ${ }^{1,2}$ Among such complications of

Received July 23, 2018. Revised January 21, 2019. Accepted January 23, 2019. Correspondence to Yuji Maehata, Department of Medicine and Clinical Science, Graduate School of Medical Sciences, Kyushu University, Maidashi 3-1-1, Higashi-ku, Fukuoka 812-8582, Japan. Tel: +81-92-642-5261, Fax: +81-92-642-5273, E-mail: ymaehata@intmed2.med.kyushu-u.ac.jp ORCID Yuji Maehata (https://orcid.org/0000-0002-7003-4615)
$\mathrm{CD}$, intestinal stricture is most common, ${ }^{3,4}$ and the prevalence of intestinal stricture has been reported to increase during the clinical course of CD. ${ }^{5}$ However, patients with $\mathrm{CD}$ and intestinal stricture are also encountered at the time of initial diagnosis, presumably due to the paucity of obstructive symptoms of such patients. It has been reported that $11 \%$ of $\mathrm{CD}$ patients already had intestinal strictures at the initial diagnosis, ${ }^{2}$ and a report by Aloi et al. ${ }^{6}$ demonstrated that patients showing the stricturing type of CD accounted for up to $36 \%$ of the cases at the time of initial diagnosis.

The recent advances in medical therapies using biologics and immunomodulators have clearly improved the clinical courses of patients with moderate to severe $\mathrm{CD}^{7-10}$ It was also 
reported that the application of biologics can decrease postoperative recurrence in CD patients. ${ }^{11-13}$ A clinical benefit of early surgical intervention in patients with the stricturing type of $\mathrm{CD}$ at the initial diagnosis thus seems possible when considering the less aggressive behavior of the stricturing type compared to the penetrating type of $\mathrm{CD},{ }^{14}$ although unnecessary intestinal resection should obviously be avoided. In this regard, the identification of the clinical characteristics associated with early surgical intervention could be useful for the selection of the appropriate candidates.

Here we retrospectively investigated the clinical course of our patients with the stricturing type of CD in order to clarify the clinical characteristics associated with early small bowel surgery after the initial diagnosis.

\section{METHODS}

\section{Patients}

This was a retrospective single-center study. During the period from 2000 and 2015, we diagnosed 196 Japanese patients with CD. The diagnosis of CD was based on clinical, radiological, endoscopic, and pathological findings using Japanese diagnostic criteria. ${ }^{15}$ Among these patients, 60 had small bowel strictures at the initial diagnosis, and we excluded 7 patients who required subsequent small bowel surgery at the time of initial diagnosis. Nine patients who had received minor surgical procedures before the diagnosis of $\mathrm{CD}$ were included for the present study (anal fistula in 5, appendectomy in 3, and inguinal hernia in a patient). Thus, the remaining 53 patients who were followed-up under treatment with medications after the initial diagnosis were analyzed.

The clinical information of the patients was extracted from the Kyushu University database of a multicenter research study on the therapeutic efficacy and prognosis of IBD. This database includes demographic data, CDAI values, laboratory data, radiologic and endoscopic findings, and medications at the time of diagnosis. This database was approved by the Institutional Review Board of Kyushu University Hospital (IRB No. 30-125) and has been registered in the University Hospital Medical Information Network Clinical Trials Registry (UMIN-CTR) as no. UMIN000017856. Written informed consent for their case to be analyzed was obtained from each patient included in the study.

\section{Assessment of Clinical Features}

We reviewed the case records of the database to obtain pati- ents' clinical variables at the diagnosis of $\mathrm{CD}$, including gender, age, smoking habit, extent of the involvement (ileitis type or ileocolitis type), disease activity, and the presence of intestinal complications. Further clinical information such as abdominal symptoms, CDAI score, ${ }^{16}$ and the serum CRP levels during the follow-up period were extracted from the patients' electronic clinical records. We also assessed medical treatments during follow-up, including elemental diet ( $\geq 900 \mathrm{kcal}$ ), and steroid, immunomodulator (azathioprine or 6-mercaptopurine), and biological therapy (infliximab or adalimumab). Medical treatments during follow-up had been selected on physicians' discretion based on the clinical symptoms, disease activities, morphologic findings, and the patients' preference.

\section{Small Bowel Stricture under Radiography}

The presence of a small bowel stricture was determined under double contrast radiography of the small intestine ${ }^{17}$ at the initial diagnosis. Briefly, the patient was prepared by an insertion of a nasojejunal tube under fluoroscopy. The tube was fixed at the ligament of Treitz by pneumodilatation of the balloon at the tip of the tube. Then, 200 to $300 \mathrm{~mL}$ of $70 \% \mathrm{v} / \mathrm{w}$ barium sulphate was slowly injected through the tube until the terminal ileum was filled with the contrast material. The small bowel was then inflated with 800 to $1,000 \mathrm{~mL}$ of air injected through the tube. When sufficient inflation was achieved, $40 \mathrm{mg}$ of scopolamine butyl bromide was injected intravenously to inhibit peristalsis and to obtain double-contrast images.

Radiographic images were retrospectively reviewed by 2 gastroenterologists (Y.M. and Y.N.), and they evaluated the presence of small bowel strictures. The presence of multiple strictures ( $\geq 5$ lesions), long-segment stricture $(>3 \mathrm{~cm})$ and prestenotic dilatation was also determined (Fig. 1). When the interpretation of radiographic findings was different between the 2 observers, they discussed with each other until the common consensus could be obtained.

\section{Study Endpoints}

The endpoint of the present study was the requirement of small bowel surgery. Small bowel surgery included any types of small bowel resection. Small bowel strictureplasty was also included in the small bowel surgery, whereas endoscopic balloon dilation and other minor surgical procedures were not counted. We investigated the relationships between small bowel surgery and the following: clinical characteristics and radiographic findings at the initial diagnosis, and types of medications used during follow-up. We also reviewed the clinical course of the 


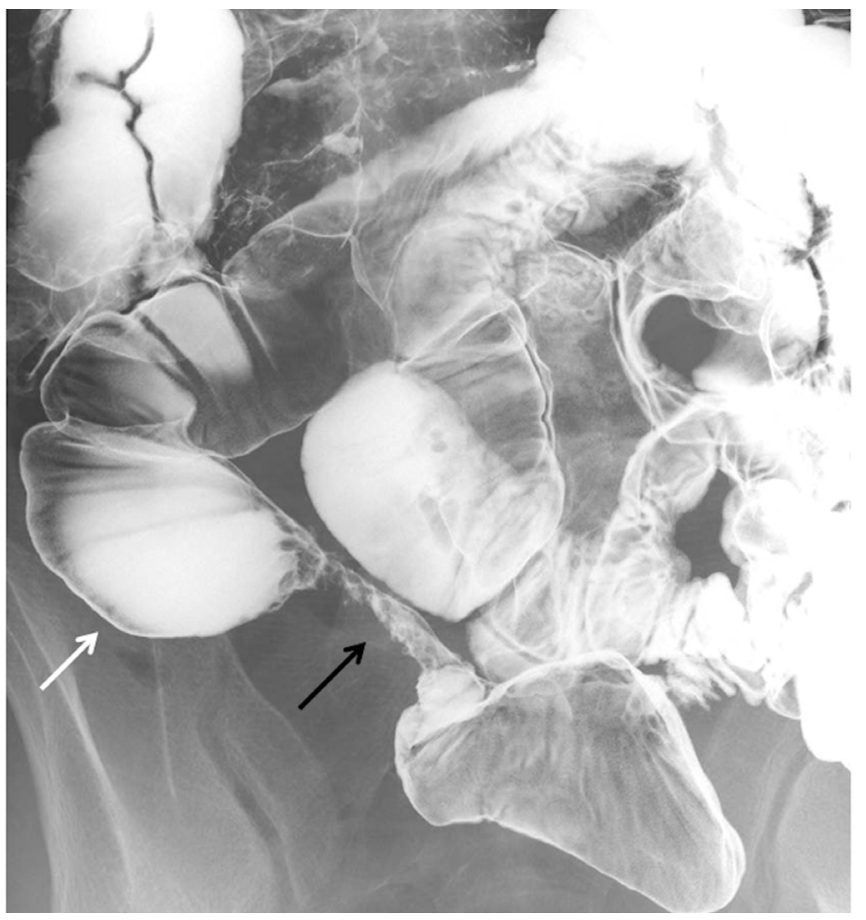

Fig. 1. Small bowel radiographic findings of long-segment stricture (black arrow) and prestenotic dilation (white arrow).

patients treated by immunomodulator or biological therapy.

\section{Statistical Analyses}

Numerical data were compared by the Mann-Whitney $U$-test, and categorical data were compared by Fisher exact probability test or the chi-square test. The cumulative incidence rate of small bowel surgery in each group was calculated by the Kaplan-Meier method, and these rates were compared using the log-rank test. To identify factors associated with small bowel surgery, we performed a multivariate analysis using the Cox proportional hazard model. A $P$-value $<0.05$ was regarded as significant for each test. All analyses were performed with the JMP Pro 12.2.0 software program (SAS Institute, Cary, NC, USA).

\section{RESULTS}

\section{Demographics}

Table 1 summarizes the demographic data of the patients at their initial diagnoses of CD. The patients were 38 males and 15 females ranging from 13 to 72 years (mean \pm SD, $30 \pm 13$ years) of age. Habitual smoking was found in 10 patients (19\%) at the time of diagnosis, 5 of whom quit smoking after the diagnosis of CD. Their serum CRP levels ranged from 0.02 to 17.50 $\mathrm{mg} / \mathrm{dL}$ (median, $2.15 \mathrm{mg} / \mathrm{dL}$ ) and their CDAI values ranged
Table 1. Baseline Characteristics at the Initial Diagnosis of $C D$ $(n=53)$

\begin{tabular}{lc}
\hline Characteristic & Value \\
\hline Male & $38(72)$ \\
Female & $15(28)$ \\
Age (yr) & $26(13-72)$ \\
Current smoking & $10(19)$ \\
Involved site & \\
Ileitis type & $27(51)$ \\
Ileocolitis type & $26(49)$ \\
CDAl & $155(21-489)$ \\
CRP (mg/dL) & $2.15(0.02-17.50)$ \\
Small bowel radiographic finding & \\
Multiple strictures, $\geq 5$ lesions & $21(40)$ \\
Long-segment stricture, $>3$ cm & $20(38)$ \\
Prestenotic dilation & $13(25)$ \\
Intestinal complication & \\
Entero-enteric fistula & $23(43)$ \\
Ileo-colonic fistula & $3(6)$ \\
Enterovesical fistula & $2(4)$ \\
Abdominal abscess & $10(19)$ \\
Obstructive symptoms & $5(9)$ \\
Anal fistula & $9(17)$ \\
Perianal abscess & \\
Medications during follow-up & \\
Elemental diet, $\geq 900$ kcal/day & \\
Steroid & \\
Immunomodulator & \\
\hline
\end{tabular}

Values are presented as number (\%) or median (range).

from 21 to 489 (median, 155). Under small bowel radiography, long-segment stricture was determined in 20 patients (38\%), and prestenotic dilation was observed in 13 patients (25\%), among whom 9 patients had obstructive symptoms. An entero-enteric, ileo-colonic or entero-vesical fistula was observed in 6 patients (10\%), and an anal fistula was identified in 6 patients (10\%).

After the initial diagnosis, an elemental diet was used in 22 patients, an immunomodulator was administered to 10 patients (19\%, azathioprine in all cases), and biological therapy was administered to 23 patients (43\%, infliximab in 19 and adalimumab in 4 ). 


\section{The Cumulative Incidence of Small Bowel Surgery and the Clinical Outcomes}

Of the 53 patients, 28 (53\%) required small bowel surgery during the median follow-up period of 5.0 years (range, $0.5-14.3$ years). We calculated the cumulative incidence of small bowel surgery at 2,5 , and 10 years as $26.4 \%, 41.0 \%$, and $63.2 \%$, respectively. The types of surgeries in 28 patients was as follows; ileocecal resection $(n=8)$, small bowel partial resection $(n=8)$, partial resection plus small bowel strictureplasty $(n=6)$, ileocecal resection plus small bowel strictureplasty $(n=3)$, ileocecal resection plus high anterior resection $(\mathrm{n}=1)$, right hemicolectomy $(\mathrm{n}=1)$, and small bowel strictureplasty alone $(\mathrm{n}=1)$. All 28 patients who underwent small bowel surgery did so due to the exacerbation of small bowel strictures. Nine of the 28 patients were accompanied by an abdominal abscess or intestinal fistula at the time of surgery.

\section{Clinical Factors Associated with Small Bowel Surgery after the Initial Diagnosis of CD}

Table 2 compares the cumulative incidence of small bowel surgery with each clinical characteristic. The univariate analysis indicated that the incidence of small bowel surgery was significantly higher in the patients with obstructive symptoms at the initial diagnosis compared to those without such symptoms $(P=0.036)$. Patients with a long-segment stricture $(P<$ $0.0001)$ (Fig. 2A) or prestenotic dilation $(P<0.0001)$ (Fig. 2B) had significantly higher incidences of small bowel surgery compared to those without these conditions.

In addition, the groups of patients receiving an immunomodulator $(P=0.037)$ or biological therapy $(P=0.008)$ (Fig. 3 ) also had significant lower incidences of small bowel surgery. Other factors, including sex, age, smoking, clinical subtype, disease activity, multiple strictures, elemental diet, and steroid were not associated with the cumulative incidence of small bowel surgery.

As shown in Table 3, the multivariate analysis revealed that long-segment stricture (HR, $4.25 ; 95 \% \mathrm{CI}, 1.78-10.53 ; P=0.001$ ) and prestenotic dilation (HR, 3.41; 95\% CI, 1.24-9.62; $P=0.018$ ) were positively associated and biological therapy (HR, 0.40; 95\% CI, 0.15-0.99; $P=0.048$ ) was negatively associated with the risk of small bowel surgery.

\section{Clinical Course of Patients Treated by Immunomodulators or Biologics}

After the diagnosis, 10 patients had been treated by azathioprine with the median duration of 6.6 years (range, 1.8-14.3
Table 2. Comparison of Cumulative Incidence of Small Bowel Surgery According to the Clinical Characteristics of the Patients at the Initial Diagnosis

\begin{tabular}{|c|c|c|}
\hline Characteristic & $\begin{array}{c}\text { 5-Year incidence rate } \\
\text { of surgery }(\%)^{a}\end{array}$ & $P$-value ${ }^{b}$ \\
\hline Sex & & 0.458 \\
\hline Male & 40.2 & \\
\hline Female & 42.9 & \\
\hline Age (yr) & & 0.308 \\
\hline$\geq 30$ & 47.4 & \\
\hline$<30$ & 35.6 & \\
\hline Current smoking & & 0.134 \\
\hline Present & 50.0 & \\
\hline Absent & 38.9 & \\
\hline Clinical subtype & & 0.953 \\
\hline Ileocolitis type & 45.8 & \\
\hline lleitis type & 37.0 & \\
\hline CDAl & & 0.876 \\
\hline$\geq 220$ & 52.4 & \\
\hline$<220$ & 36.9 & \\
\hline $\mathrm{CRP}(\mathrm{mg} / \mathrm{dL})$ & & 0.853 \\
\hline$\geq 3$ & 41.5 & \\
\hline$<3$ & 40.4 & \\
\hline Obstructive symptoms & & 0.036 \\
\hline Present & 66.7 & \\
\hline Absent & 35.6 & \\
\hline Multiple strictures & & 0.623 \\
\hline Present & 38.1 & \\
\hline Absent & 43.1 & \\
\hline Long-segment stricture & & $<0.0001$ \\
\hline Present & 79.0 & \\
\hline Absent & 18.9 & \\
\hline Prestenotic dilation & & $<0.0001$ \\
\hline Present & 88.5 & \\
\hline Absent & 26.4 & \\
\hline Elemental diet & & 0.841 \\
\hline Present & 37.9 & \\
\hline Absent & 42.9 & \\
\hline Steroid & & 0.248 \\
\hline Present & 33.3 & \\
\hline Absent & 41.9 & \\
\hline Immunomodulator & & 0.037 \\
\hline Present & 22.9 & \\
\hline Absent & 45.0 & \\
\hline Biological therapy & & 0.008 \\
\hline Present & 24.8 & \\
\hline Absent & 53.6 & \\
\hline
\end{tabular}

Values were estimated by Kaplan-Meier method.

Values among the groups were compared by using the log-rank test. 

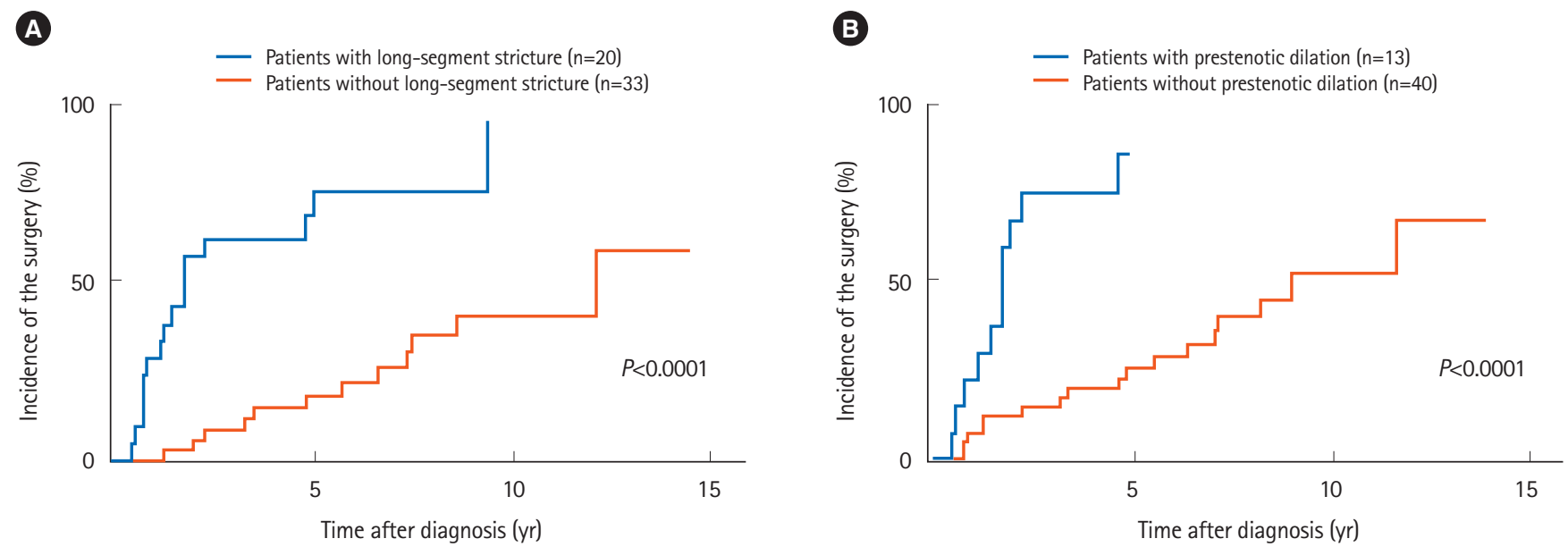

Fig. 2. Comparison of the cumulative incidence of small bowel surgery according to (A) long-segment stricture and (B) prestenotic dilation under radiography.

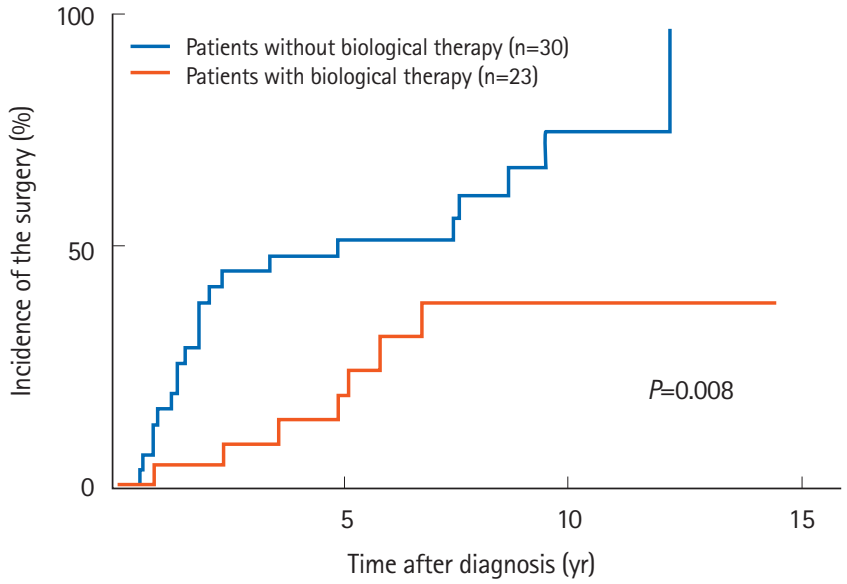

Fig. 3. Comparison of the cumulative incidence of small bowel surgery according to biological therapy.

years) and 23 patients had been treated by biological therapy with the median duration of 5.5 years (range, $0.8-14.3$ years). Among them, 6 patients had been treated with the combination of azathioprine and biological therapy. Of the 10 patients taking azathioprine, 2 patients (20\%) required small bowel surgery at 1.8 years and 5 years after the diagnosis. Azathioprine was withdrawn 4 years after the diagnosis in a patient because of the adverse effect. Of the 23 patients treated by biological therapy, 7 patients (30\%) underwent small bowel surgery at the median duration of 4.8 years (range, $0.8-6.6$ years) after the diagnosis. While biological therapy was switched to another one in 4 patients because of the loss of response to the biological therapy, small bowel surgery could be avoided in 16 patients during follow-up.
Table 3. Risk of Small Bowel Surgery Shown by the Multivariate Analysis

\begin{tabular}{lcc}
\hline & HR $(95 \% \mathrm{Cl})$ & $P$-value \\
\hline Long-segment stricture & $4.25(1.78-10.53)$ & 0.001 \\
Prestenotic dilation & $3.41(1.24-9.62)$ & 0.018 \\
Biological therapy & $0.40(0.15-0.99)$ & 0.048 \\
Immunomodulator & $0.33(0.05-1.17)$ & 0.091 \\
Obstructive symptoms & $0.95(0.36-2.28)$ & 0.907 \\
\hline
\end{tabular}

\section{DISCUSSION}

We evaluated the clinical course of 53 patients with the stricturing type of $\mathrm{CD}$ at the initial diagnosis to investigate clinical factors associated with subsequent small bowel surgery. The results of our analyses demonstrated that a long-segment small bowel stricture and prestenotic dilation under radiography and obstructive symptoms at the initial diagnosis were positively associated with small bowel surgery, and that biological therapy was negatively associated with small bowel surgery.

According to the Montreal classification, the stricturing phenotype of CD is defined as the presence of constant luminal narrowing with prestenotic dilation and/or obstructive symptoms. ${ }^{18}$ However, the length of small bowel strictures was not clearly defined in previous studies. ${ }^{18,19}$ In the present study, a long-segment $(>3 \mathrm{~cm})$ stricture under small bowel radiography was revealed as an independent factor associated with early surgical intervention. Since a long-segment $(>3 \mathrm{~cm})$ stricture has been reported to be a poor predictor of successful endoscopic balloon dilation, ${ }^{20}$ the length of a small bowel stric- 
ture as well as the presence of prestenotic dilation and obstructive symptoms should be considered when the indications for small bowel surgery are being considered.

Previous studies examined the association between medical treatments and the risk of surgery in patients with $\mathrm{CD}^{21-28}$ Among the medications, immunomodulators were negatively associated with the risk of small bowel surgery in the present study's univariate analysis. It was recently indicated that treatment with azathioprine, especially the very early use of the medication, is associated with the reduced risk of surgical resection, ${ }^{23-25}$ and we thus speculate that our results imply a preventive effect of azathioprine against early surgical intervention in patients with $\mathrm{CD}$ of the stricturing type.

We observed that biological therapy with infliximab or adalimumab was also negatively associated with early surgical intervention in the present series. Although it was recently suggested that these medications can alter the natural history and decrease the risk of surgical intervention in CD patients, ${ }^{26-28}$ the indications for biological therapy for patients with stricturing-type $\mathrm{CD}$ remain controversial ${ }^{29-31}$ because rapid luminal healing achieved with this therapy can exacerbate small bowel strictures. ${ }^{19}$ Although the indications for early biological intervention remain uncertain and the stricturing type is not considered a risk factor of disabling $\mathrm{CD},{ }^{32,33}$ the stricturing type of $\mathrm{CD}$ at the initial diagnosis can be a possible indication for early biological intervention. However, considering the potential bias related to the retrospective nature of the present study, further evaluations in prospective studies are needed.

The present study has several limitations. First, we assessed small bowel strictures by using small bowel radiography findings, which could possibly misdiagnose the number of small bowel strictures in patients with $\mathrm{CD}^{3}{ }^{34,35}$ In this sense, CT enterography or magnetic resonance enterography might be more appropriate. ${ }^{36}$ However, the severity and the length, but not the number, of small bowel strictures was associated with early surgical intervention in the present patients with stricturingtype $\mathrm{CD}$. In addition, magnetic resonance enterography might not be suitable for the evaluation of small bowel strictures in $\mathrm{CD}^{37}$ Therefore, we believe that this limitation did not hamper our findings. Second, this was a single-center retrospective study with a small number of patients without standard protocol for decision making the therapy during follow-up. Nevertheless, because no investigation focusing on CD patients with complicating small bowel stenosis at the initial diagnosis has been reported to date, our present results can be considered to have some clinical impact on the management of CD. Third, it could cause potential bias in the evaluation of radiographic findings because 1 observer was not blinded to the demographic data of the study subjects. In order to reduce the risk of potential bias, the order of radiography of the patients was shuffled when evaluating their images.

In conclusion, our analyses demonstrated that early small bowel surgery appears to be an appropriate choice of therapy for patients with CD and a long-segment stricture or prestenotic dilation at the initial diagnosis. In patients with milder small bowel strictures, biological therapy can be helpful, but further studies must be conducted to establish the early indications of such medications.

\section{FINANCIAL SUPPORT}

The authors received no financial support for the research, authorship, and/or publication of this article.

\section{CONFLICT OF INTEREST}

No potential conflict of interest relevant to this article was reported.

\section{AUTHOR CONTRIBUTION}

Study concept and design: Maehata Y and Nagata Y. Acquisition of data: Maehata Y, Nagata Y, Moriyama T, Matsuno Y, Hirano A, Umeno J, Torisu T, and Manabe T. Writing-original draft: Maehata Y. Writing-review and editing: Kitazono T and Esaki M. Critical revision of the manuscript for important intellectual content: Kitazono T and Esaki M. Approval of final manuscript: all authors.

\section{REFERENCES}

1. Baumgart DC, Sandborn WJ. Crohn's disease. Lancet 2012; 380:1590-1605.

2. Louis E, Collard A, Oger AF, Degroote E, Aboul Nasr El Yafi FA, Belaiche J. Behaviour of Crohn's disease according to the Vienna classification: changing pattern over the course of the disease. Gut 2001;49:777-782.

3. Cleynen I, González JR, Figueroa C, et al. Genetic factors conferring an increased susceptibility to develop Crohn's disease also influence disease phenotype: results from the IBDchip European Project. Gut 2013;62:1556-1565.

4. Yano Y, Matsui T, Hirai F, et al. Cancer risk in Japanese Crohn's 
disease patients: investigation of the standardized incidence ratio. J Gastroenterol Hepatol 2013;28:1300-1305.

5. Cosnes J. Crohn's disease phenotype, prognosis, and long-term complications: what to expect? Acta Gastroenterol Belg 2008; 71:303-307.

6. Aloi M, Viola F, D'Arcangelo G, et al. Disease course and efficacy of medical therapy in stricturing paediatric Crohn's disease. Dig Liver Dis 2013;45:464-468.

7. Hanauer SB, Feagan BG, Lichtenstein GR, et al. Maintenance infliximab for Crohn's disease: the ACCENT I randomised trial. Lancet 2002;359:1541-1549.

8. Hanauer SB, Sandborn WJ, Rutgeerts P, et al. Human anti-tumor necrosis factor monoclonal antibody (adalimumab) in Crohn's disease: the CLASSIC-I trial. Gastroenterology 2006; 130:323-333.

9. Matsumoto T, Iida M, Motoya S, et al. Therapeutic efficacy of infliximab on patients with short duration of Crohn's disease: a Japanese multicenter survey. Dis Colon Rectum 2008;51:916923.

10. Colombel JF, Sandborn WJ, Reinisch W, et al. Infliximab, azathioprine, or combination therapy for Crohn's disease. N Engl J Med 2010;362:1383-1395.

11. Regueiro M, Kip KE, Baidoo L, Swoger JM, Schraut W. Postoperative therapy with infliximab prevents long-term Crohn's disease recurrence. Clin Gastroenterol Hepatol 2014;12:14941502.

12. De Cruz P, Kamm MA, Hamilton AL, et al. Crohn's disease management after intestinal resection: a randomised trial. Lancet 2015;385:1406-1417.

13. Regueiro M, Feagan BG, Zou B, et al. Infliximab reduces endoscopic, but not clinical, recurrence of Crohn's disease after ileocolonic resection. Gastroenterology 2016;150:1568-1578.

14. Samimi R, Flasar MH, Kavic S, Tracy K, Cross RK. Outcome of medical treatment of stricturing and penetrating Crohn's disease: a retrospective study. Inflamm Bowel Dis 2010;16:11871194.

15. Yao T, Matsui T, Hiwatashi N. Crohn's disease in Japan: diagnostic criteria and epidemiology. Dis Colon Rectum 2000;43 (10 Suppl):S85-S93.

16. Best WR, Becktel JM, Singleton JW, Kern F Jr. Development of a Crohn's disease activity index. National Cooperative Crohn's Disease Study. Gastroenterology 1976;70:439-444.

17. Matsumoto T, Esaki M, Kurahara K, et al. Double-contrast barium enteroclysis as a patency tool for nonsteroidal anti-inflammatory drug-induced enteropathy. Dig Dis Sci 2011;56:32473253.
18. Satsangi J, Silverberg MS, Vermeire S, Colombel JF. The Montreal classification of inflammatory bowel disease: controversies, consensus, and implications. Gut 2006;55:749-753.

19. Rieder F, Zimmermann EM, Remzi FH, Sandborn WJ. Crohn's disease complicated by strictures: a systematic review. Gut 2013;62:1072-1084.

20. Hirai F, Beppu T, Sou S, Seki T, Yao K, Matsui T. Endoscopic balloon dilatation using double-balloon endoscopy is a useful and safe treatment for small intestinal strictures in Crohn's disease. Dig Endosc 2010;22:200-204.

21. Lakatos PL, Golovics PA, David G, et al. Has there been a change in the natural history of Crohn's disease? Surgical rates and medical management in a population-based inception cohort from Western Hungary between 1977-2009. Am J Gastroenterol 2012;107:579-588.

22. Fu YT, Hong T, Round A, Bressler B. Impact of medical therapy on patients with Crohn's disease requiring surgical resection. World J Gastroenterol 2014;20:11808-11814.

23. Chhaya V, Pollok RC, Cecil E, et al. Impact of early thiopurines on surgery in 2770 children and young people diagnosed with inflammatory bowel disease: a national population-based study. Aliment Pharmacol Ther 2015;42:990-999.

24. Cosnes J, Bourrier A, Laharie D, et al. Early administration of azathioprine vs conventional management of Crohn's disease: a randomized controlled trial. Gastroenterology 2013;145:758765.

25. Chatu S, Subramanian V, Saxena S, Pollok RC. The role of thiopurines in reducing the need for surgical resection in Crohn's disease: a systematic review and meta-analysis. Am J Gastroenterol 2014;109:23-34.

26. Feagan BG, Panaccione R, Sandborn WJ, et al. Effects of adalimumab therapy on incidence of hospitalization and surgery in Crohn's disease: results from the CHARM study. Gastroenterology 2008;135:1493-1499.

27. Schnitzler F, Fidder H, Ferrante M, et al. Long-term outcome of treatment with infliximab in 614 patients with Crohn's disease: results from a single-centre cohort. Gut 2009;58:492-500.

28. Rungoe C, Langholz E, Andersson M, et al. Changes in medical treatment and surgery rates in inflammatory bowel disease: a nationwide cohort study 1979-2011. Gut 2014;63:1607-1616.

29. Lichtenstein GR, Olson A, Travers S, et al. Factors associated with the development of intestinal strictures or obstructions in patients with Crohn's disease. Am J Gastroenterol 2006;101: 1030-1038.

30. Pelletier AL, Kalisazan B, Wienckiewicz J, Bouarioua N, Soulé JC. Infliximab treatment for symptomatic Crohn's disease stric- 
tures. Aliment Pharmacol Ther 2009;29:279-285.

31. Condino G, Calabrese E, Zorzi F, et al. Anti-TNF-alpha treatments and obstructive symptoms in Crohn's disease: a prospective study. Dig Liver Dis 2013;45:258-262.

32. Gomollón F, Dignass A, Annese V, et al. 3rd European evidence-based consensus on the diagnosis and management of Crohn's disease 2016. Part 1: diagnosis and medical management. J Crohns Colitis 2017;11:3-25.

33. Matsuoka K, Kobayashi T, Ueno F, et al. Evidence-based clinical practice guidelines for inflammatory bowel disease. J Gastroenterol 2018;53:305-353.

34. Otterson MF, Lundeen SJ, Spinelli KS, et al. Radiographic underestimation of small bowel stricturing Crohn's disease: a comparison with surgical findings. Surgery 2004;136:854-860.

35. Angriman I, Scarpa M, Ruffolo C, et al. Double contrast smallbowel radiography in the preoperative assessment of Crohn's disease: is it still useful? Surg Today 2008;38:700-704.

36. Greenup AJ, Bressler B, Rosenfeld G. Medical imaging in small bowel Crohn's disease-computer tomography enterography, magnetic resonance enterography, and ultrasound: "Which one is the best for what?". Inflamm Bowel Dis 2016;22:12461261.

37. Takenaka K, Ohtsuka K, Kitazume Y, et al. Comparison of magnetic resonance and balloon enteroscopic examination of the small intestine in patients with Crohn's disease. Gastroenterology 2014;147:334-342. 